\title{
Fuzzy Logic in Sports: A Review and an Illustrative Case Study in the Field of Strength Training
}

\author{
Hristo Novatchkov \\ Centre for Sport Science and University Sports \\ University of Vienna \\ Auf der Schmelz 6a
}

\author{
Arnold Baca \\ Centre for Sport Science and University Sports \\ University of Vienna \\ Auf der Schmelz 6a
}

\begin{abstract}
As a special form of probabilistic reasoning, the fuzzy logic concept allows the effective realization of approximate, vague, uncertain, dynamic, continuous and, at the same time, more realistic conditions, which are closer to the actual physical world and human thinking. This many-valued idea involves the definition of fuzzy sets and rules as well membership functions. These techniques allow the mapping of classes of objects not only - according to the binary logic to false (0) and true (1) but also to intermediate values in between. Based on this theorem, the particular purpose of this paper was to propose a fuzzy logic approach for the evaluation of strength training exercises. The motivation for the present study arose from previous research done in the area of artificial intelligence (AI) in sports, the effective number of multidisciplinary solutions integrating fuzzy logic methodologies and the lack of applications in the fields of sport and especially strength training. The conception takes into account gathered data from sensor-equipped machines as well as recommended suggestions and criteria regarding a proper execution. The final aim is to integrate the designed procedures into a computer-based coaching framework, returning automated feedback on the performed technique.
\end{abstract}

\section{General Terms}

Sports, Intelligence, Modeling, Probabilistic Reasoning

\section{Keywords}

Strength Training, Fuzzy Logic, Evaluation, Feedback, Coaching

\section{INTRODUCTION}

In general, the fuzzy logic concept involves the idea of a vague rather than exact probabilistic reasoning with different degrees of truth. Basic ideas on many-valued logic systems were already investigated by Łukasiewicz [1] in 1920, who proposed the use of three-valued logic by adding the indeterminate condition in addition to false and true. The first mentioning of the term fuzzy logic, however, dates back to the year 1965, when Zadeh published a couple of scientific research papers in which he introduced the so-called fuzzy sets as an effective tool for the definition of more realistic classes of objects taken from real life (e.g. animals) [2, 3]. In particular, the author suggested the use of membership functions and set of rules in order to be also able to represent ambiguous states (e.g. bacteria in the class of animals) by mapping them to intermediate (rather than binary) values between 0 and 1 . In this way, this theorem can be seen as an enhancement of the boolean algebra [4], consisting of more than just two truth values.
The major goal of the present research was to apply the idea of fuzzy logic and vague states for the evaluation and classification of exercises performed on sensor-equipped weight machines in respect to continuous strength training recommendations and criteria.

\section{RELATED WORK}

Fuzzy logic methods have been increasingly introduced for a variety of purposes and application fields including modeling, data mining, decision making, expert systems, prediction, forecasting, computer vision, image processing, pattern recognition and robotics. In the area of sport, the use of fuzzy logic techniques is still a rather new but, at the same time, upcoming field of activity. However, as the following literature review shows, procedures based on the idea of uncertainty have not yet been investigated in the field of strength training.

One of the early fuzzy research interests in sports involves the implementation of intelligent decision systems for choosing a proper strategy in the game of pool $[5,6,7]$. The realization by Riley [8], on the other hand, applies continuous inference mechanisms for the purpose of scoring a goal in soccer simulation environments.

Other application areas include the domain of video processing. A generic fuzzy scheme for the semantic characterization and classification of sport videos such as sequences from games like football, cricket and volleyball is presented in [9]. The study by Refaey et al. [10] illustrates the successful use of fuzzy logic methods to identify concurrent transitions and detect shots in football videos.

Specific investigations propose the development of expert systems based on vague conditions for the analysis of fast bowling in cricket $[11,12]$. Similarly, Curtis' scientific papers suggest a training system integrating Zadeh's theory for the classification of batting strokes [13, 14]. The study by Singh et al. [15] focuses on the evaluation of performances of cricket players based on a number of approximate input parameters. The implementation calculates the ranking of the athletes for different scenarios.

Modeling based on fuzzy techniques is another common procedure, which is, for instance, used to measure daily activity $[16,17]$. A muscle model and control simulation for approximating dynamical behavior of motor units is presented by Heller and Witte [18]. In other areas such as swimming, the design of fuzzy-based machine learning algorithms aims at the modeling of sport training [19]. 
As yet another example, kinematic data is utilized in combination with many-valued algorithms to recognize movements such as different giant swings [20]. Other approaches concentrate on the identification of sport talents via an implemented web platform, integrating ambiguous expert knowledge and suggesting the most suitable discipline for a certain child [21].

\section{PROPOSED WORK}

\subsection{Background}

The present idea and work originated from previous studies done in the area of strength training $[22,23]$. In particular, research focused on the implementation of artificial intelligence (AI) techniques such as machine learning algorithms on the basis of neural networks (NNs) for the automated classification of sensor information gathered from weight training equipment. More precisely, measured parameters included way and force data characteristics collected (at a sampling rate of $100 \mathrm{~Hz}$ ) during exercises on a leg press machine (see Figure 1) equipped with a rotary encoder (DP18, Altmann) and a load cell (PW10A, Hottinger Baldwin).

Figure 2 demonstrates a comparative example of the gathered force and weight displacement data of 2 consecutive repetitions performed by the same athlete on the leg press machine. As shown, the execution in sub chart (a) is clearly identified by an instable technique with fluctuating time, force and displacement characteristics. Sub chart (b), on the other hand, illustrates a stable performance with smooth and constant sensor output. Such distinctive features were thereupon applied for the development of AI and particularly $\mathrm{NN}$ modeling methods, allowing an automated evaluation of the gathered data.

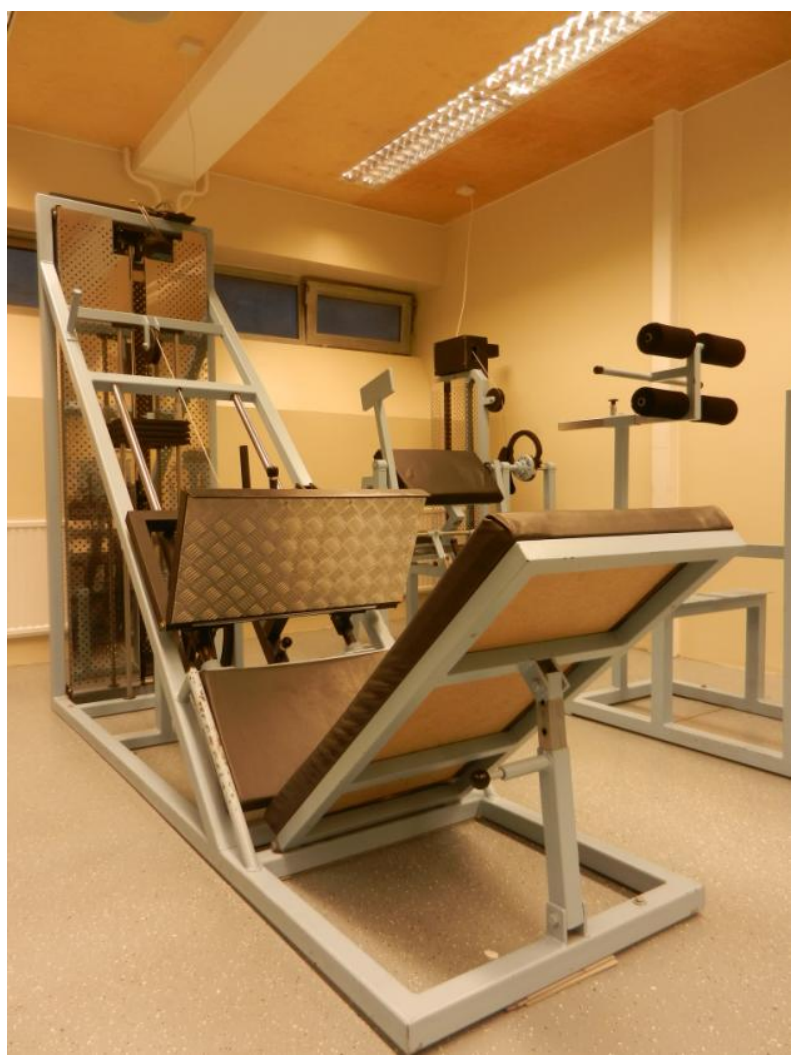

Fig 1: Leg press machine equipped with way and force sensors

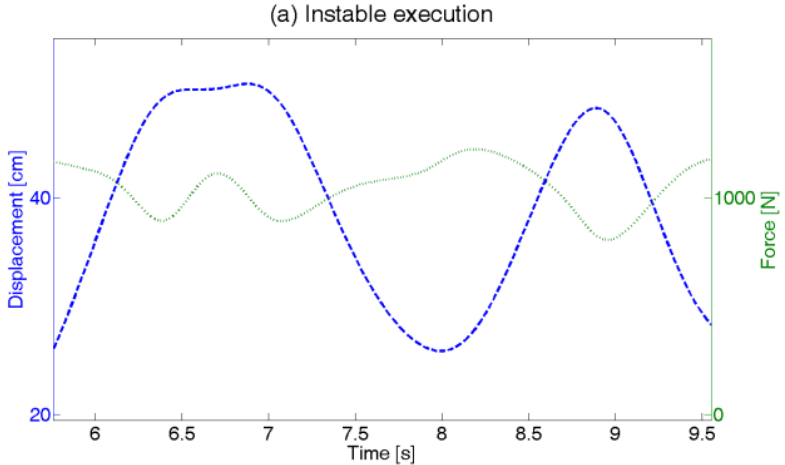

(b) Stable execution

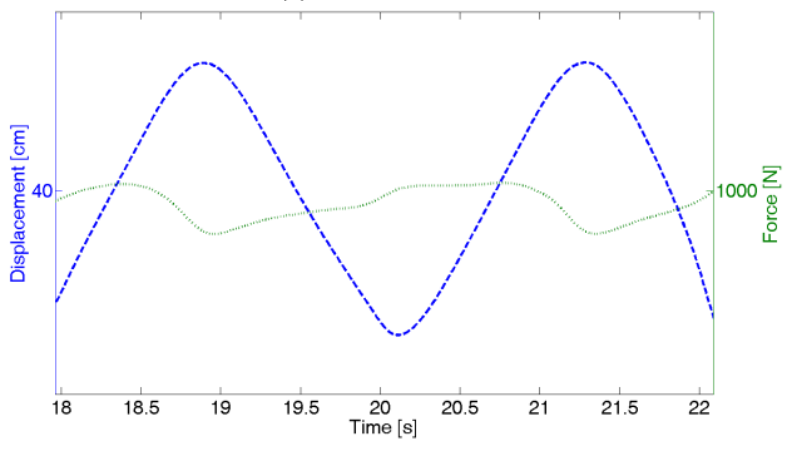

Fig 2: Comparison of instable (a) and stable (b) executions by the same athlete

Based on the obtained results with high classification and performance outcomes as well as drawn conclusions, it was implied to design fuzzy methodologies as another suitable (yet more elementary but still effective) alternative for the assessment of performed executions. The reason therefore lay also in the appropriate circumstances and applicability of vague conditions for the analysis of the used technique by the involvement of professional coaches. Similarly to the previous research, also in this study the evaluation criteria included common strength training recommendations such as time, constancy and completeness [24, 25, 26], which can be rated on the basis of deduced parameters like duration, displacement or force and velocity (depicted in the following). Such determinants can be automatically estimated by the implementation of appropriate segmentation procedures and calculations, dividing the data into single repetitions as well as extension, flexion and holding phases [23].

\subsection{Design Methodology}

The present research suggests the design of a fuzzy inference system (FIS) mapping given input data regarding the execution of the flexion and extension phases into an output value determining the overall rating of the technique.

All the development work was carried out in MATLAB ${ }^{\circledR}$ version R2010b for Windows using the so-called Fuzzy Logic Toolbox ${ }^{\mathrm{TM}}$, which provides routines for the realization, visualization and simulation of FISs. The interference type was based on the more general Mamdani method, due to its advantages of intuitive formation, widespread acceptance and suitability to human input [27].

Figure 3 illustrates a feasible example regarding the realization of a FIS in strength training. As shown, 3 different input parameters concerning the eccentric and concentric actions (in respect to the specified criteria) were defined: 


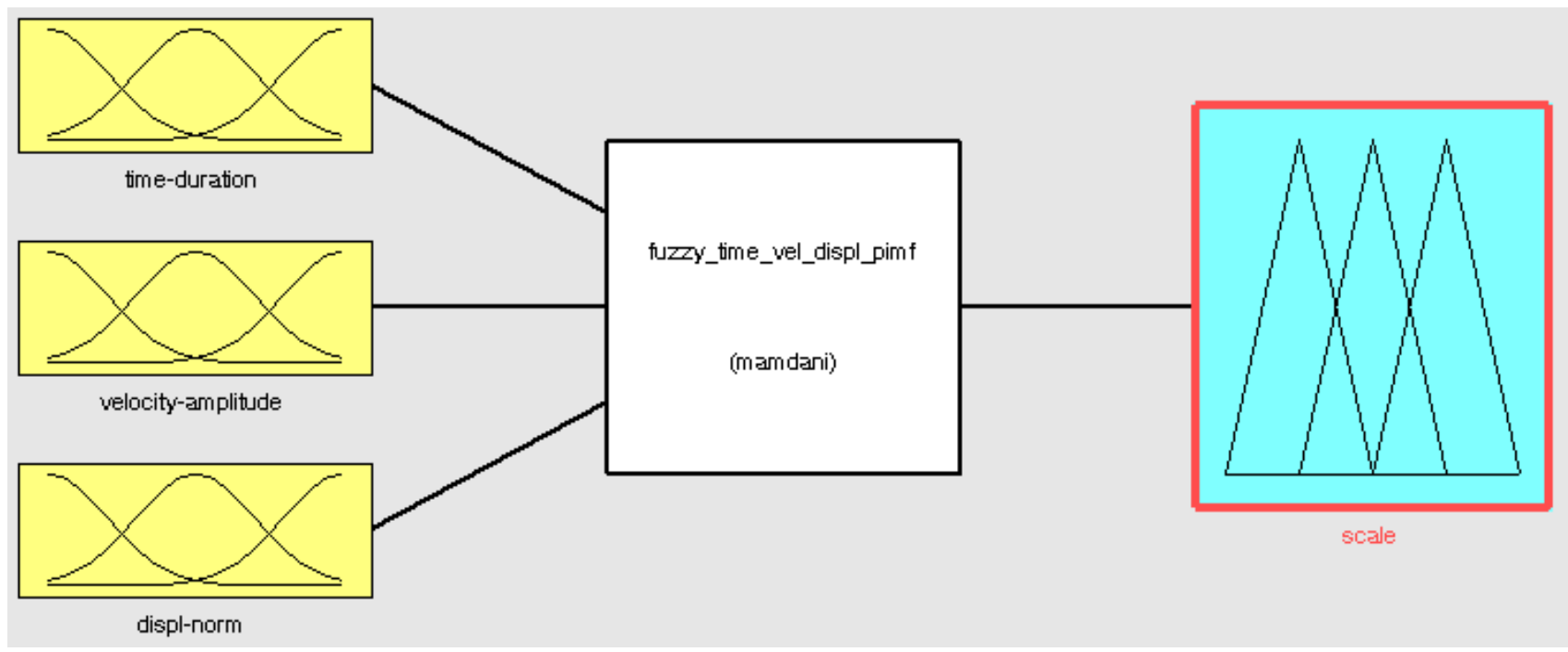

Fig 3: Feasible FIS in strength training with 3 input and 1 output parameters

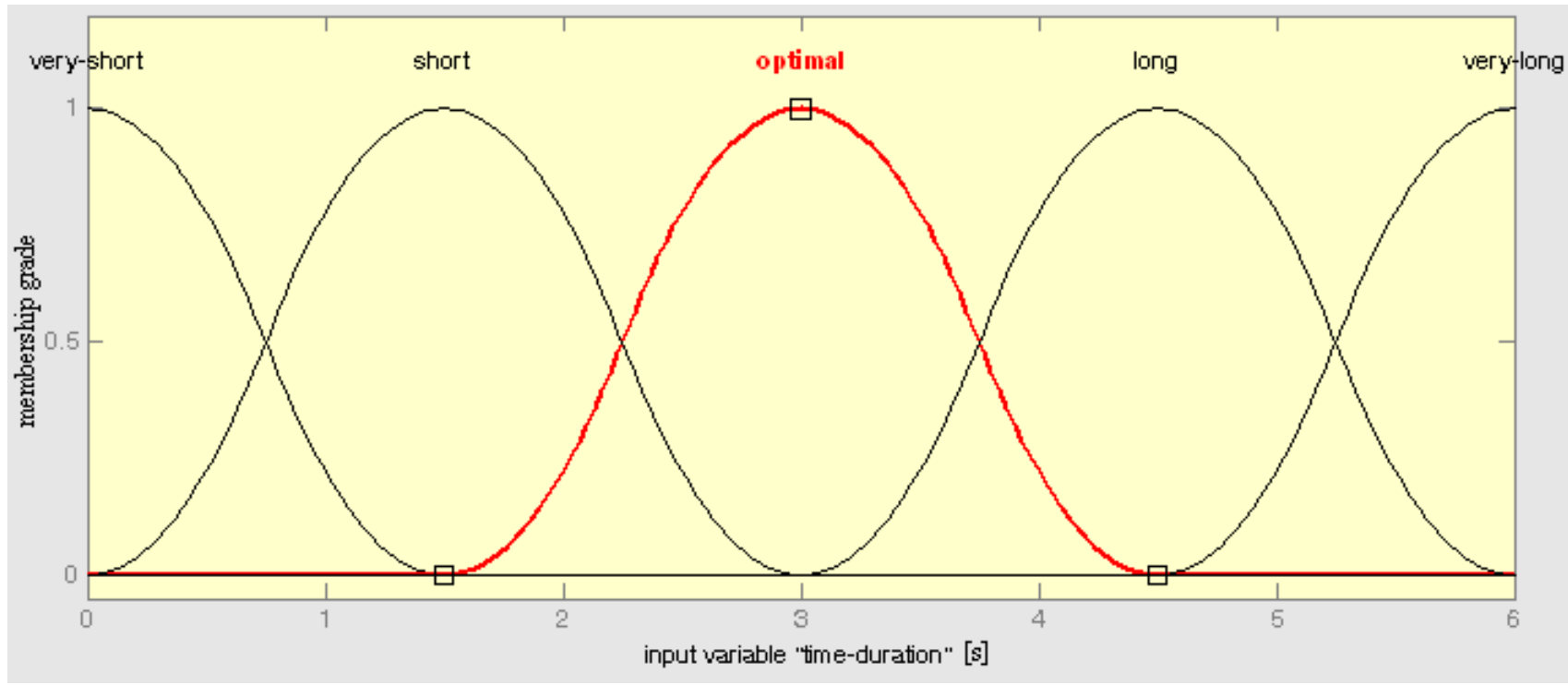

Fig 4: Exemplary fuzzy sets for the input variable mapping the time duration to a value between 0 (improper) and 1 (proper)

1. Duration (time)

2. Amplitude between the minimal and maximal velocity values (constancy)

3. Normalized displacement (completeness)

Further details on these determinants, their realization in the FIS and their specification on the basis of the definition of membership functions are described in the following section.

\subsection{Input and Output Parameters and their Membership Functions}

The time duration is a crucial determinant for the efficiency of the exercise. In general, for beginners and elderly as well as for health and injury prevention purposes, it is suggested to perform each repetition in a slow manner with a moderate speed and an approximate duration of 6 seconds per repetition [24] or 2-3 seconds for the flexion and extension [25]. These recommendations were realized in form of 5 fuzzy sets (very short, short, optimal, long and very long), mapping the time number of fuzzy sets (such as very short, short, optimal, long and very long). In this case, the displacement variable has to duration to the according degree of membership (from 0 to 1 ). Figure 4 illustrates the representation of this input variable.

As already mentioned, besides the time factor, constancy plays a second important role regarding the quality of the execution. One realization alternative for the stability in terms of a fuzzy set implementation might, for instance, focus on its representation on the basis of the performed velocity, which can be deduced from the measured sensor signals. A particular approach could involve the determination of the velocity amplitude representing the difference between the minimal and maximal velocity values of the extension and flexion phases. This solution was realized by the definition of 3 fuzzy sets (low, high and very high velocity amplitude), where a lower amplitude (in $\mathrm{m} / \mathrm{s}$ ) means a more constant execution.

The third significant criterion refers to the completeness of the exercises. Based on the gathered weight displacement values, one direct representation might integrate these signals into a be normalized accordingly - for example by the height and leg length of the individuals. A second opportunity would be 
to take into consideration the appropriate weight configuration of the leg press machine. Another practical mapping method is presented in the discussion section.

Figure 5 illustrates the implemented relation between the variables representing the constancy and completeness of the execution and the according scale values between 0 (improper) and 1 (proper). The purpose of the output variable, on the other hand, is to map the 3 input parameters into an overall rating on the basis of the constructed fuzzy rules. Figure 6 demonstrates an exemplary output configuration integrating 5 different categorizations (improper, poor, average, good and proper).

In general, the membership functions for all input and output parameters were defined by spline-based $\Pi$-shaped curves (named after their appearance). This type was chosen due to the narrow peak with a relatively high degree of membership within an acceptable limit, and gradual fall afterwards, which appeared to represent the data the best. In addition, such functions have been recently successfully applied to solve challenging classification tasks based on the advantages of easier adaptation and hence flexibility and generalization in respect to the requirements of the problem $[28,29]$.

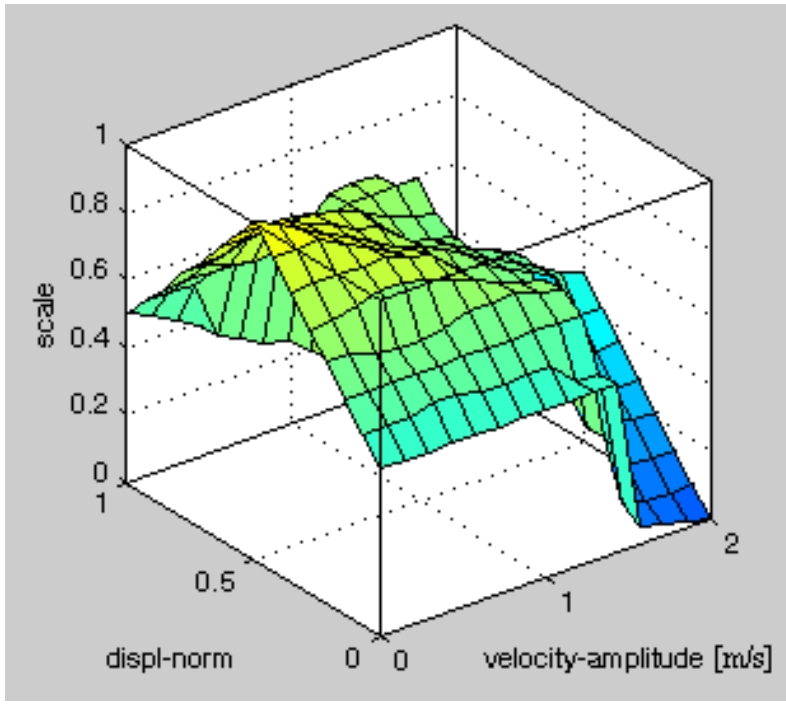

Fig 5: Relation between velocity amplitude, normalized displacement and respective scale values

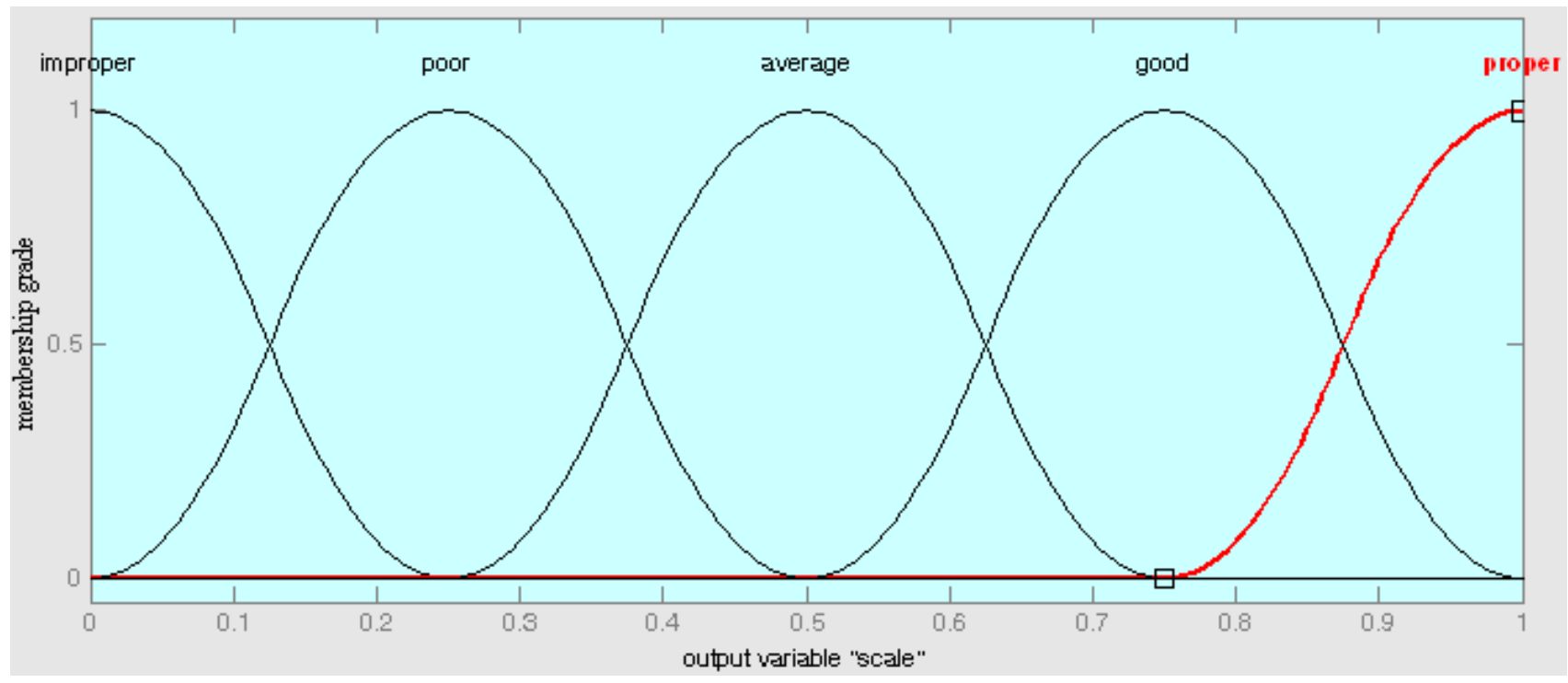

Fig 6: Exemplary fuzzy sets for output variable representing the overall rating (scale)

\subsection{Fuzzy Rules}

Based on the description of the input and output parameters, 70 consequential rules were established for mapping purposes. In the following, some of the most illustrative rules (including the specified weights), which are also taken for the subsequent simulation scenario, are depicted:

Rule 12: If (time-duration is optimal) and (velocity-amplitude is low) and (displ-norm is complete) then (scale is proper) (1)

Rule 14: If (time-duration is optimal) and (velocity-amplitude is low) and (displ-norm is short) then (scale is good) (1)

Rule 17: If (time-duration is optimal) and (velocity-amplitude is high) and (displ-norm is complete) then (scale is good) (1)

Rule 19: If (time-duration is optimal) and (velocity-amplitude is high) and (displ-norm is short) then (scale is good) (0.75)
Rule 57: If (time-duration is short) and (velocity-amplitude is low) and (displ-norm is complete) then (scale is good) (1)

Rule 59: If (time-duration is short) and (velocity-amplitude is low) and (displ-norm is short) then (scale is good) (0.75)

Rule 62: If (time-duration is short) and (velocity-amplitude is high) and (displ-norm is complete) then (scale is good) (0.75)

Rule 64: If (time-duration is short) and (velocity-amplitude is high) and (displ-norm is short) then (scale is average) (1)

\subsection{Practical Scenario}

Figure 7 illustrates a feasible scenario for a rather good execution resulting in an overall rating of 0.9. It furthermore shows the chosen parameter for each input variable as well as the most significant rules (depicted in the previous section), which affect the actual outcome. The gathered surface (lower 
right corner) demonstrates the aggregation process combining the outputs of each rule into a single fuzzy set.

The final score was calculated on the basis of the defuzzification method using the "mean of maximum" technique, which is commonly applied in combination with the Mamdani inference type. The benefit of this method is that it "selects the typical value of the terms that is most valid, rather than balancing out the different inference results" [27]. Consequently, the determined scale output in the illustrated scenario returns an exact and plausible value, which represents the resulting area the best, providing an overall rating of the performance and giving hope for the integration of such fuzzy logic implementations into immediate feedback routines and control systems in strength training.
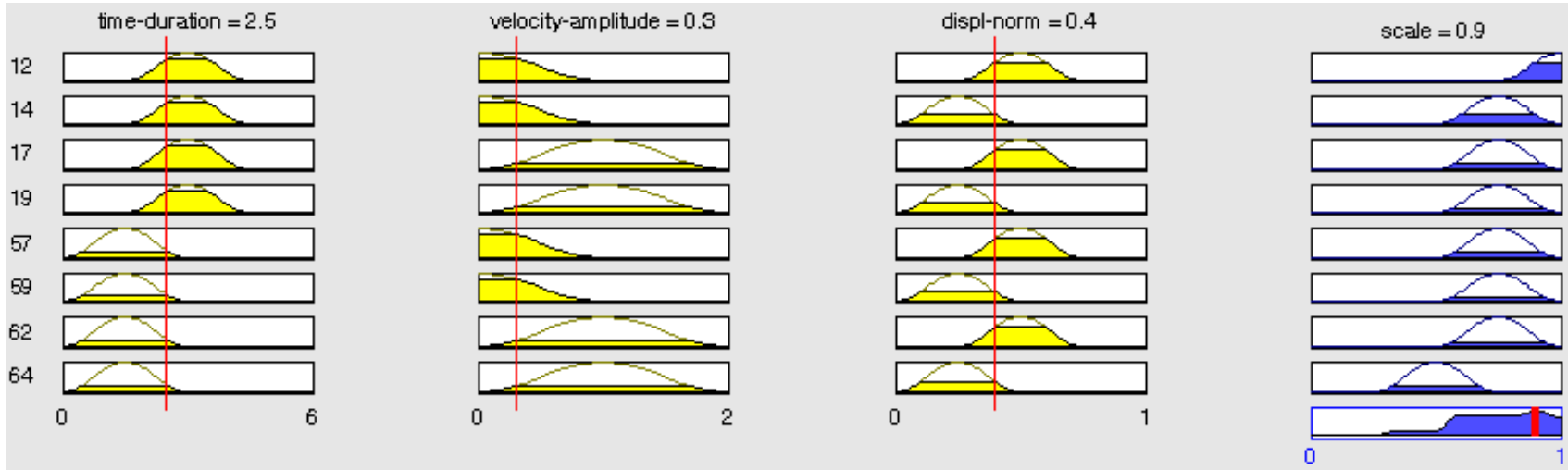

Fig 7: Feasible scenario for a rather good execution with an overall rating of 0.9

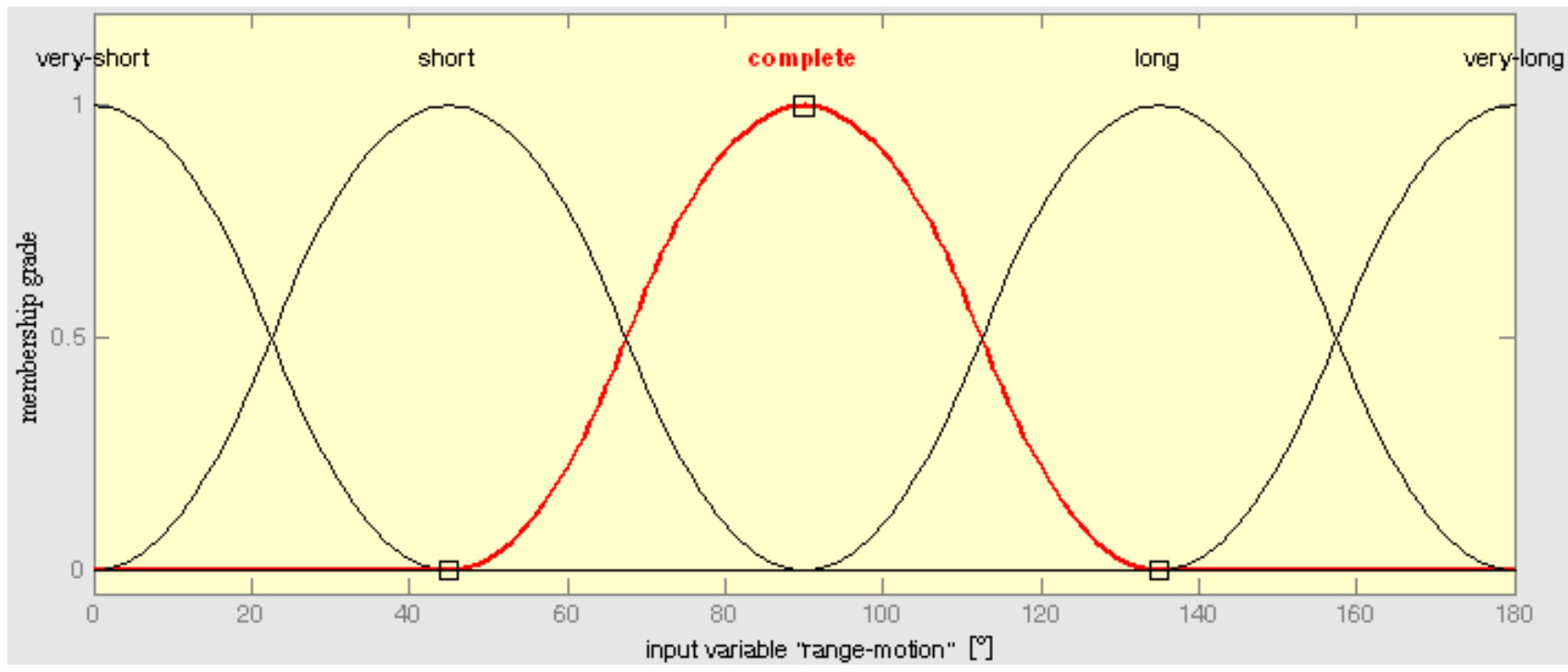

Fig 8: Exemplary fuzzy sets for input variable representing the range of motion

\section{DISCUSSION}

Fuzzy logic provides effective techniques for the representation of uncertainty and is therefore often used for the handling of vague conditions in a variety of application areas. In the field of sport, Zadeh's proposal from 1965 is still in a nascent stage, being applied in robotics, video processing, modeling and simulation, but also for the analysis and classification of sport-specific executions such as strokes in cricket.

The present paper suggests the design of fuzzy logic techniques for the evaluation of exercises performed on weight machines equipped with way and force sensors. The realization takes into account different essential assessment criteria and maps them to a cumulative scale, on the one hand. In addition to the accumulated rating, however, it is also possible to determine specific abnormalities such as occurred mistakes by considering the defined input variables including the specified fuzzy sets and respective membership functions. In this way, feedback on the overall execution as well on particular factors and characteristics can be given, contributing to the diversity of the approach. In this regard, other major advantages of the presented fuzzy logic idea are also the flexibility and simple adaptation facilities to various strength recommendations like, for example, specific training programs for muscle hypertrophy.

Another benefit of the conceptual design is that there is no necessity of large training data in comparison to other applied classification procedures such as NN modeling. In general, this kind of machine learning techniques require large volume of input information covering sufficient variations of possible situations. Consequently, more computing time is needed for building the model, while, at the same time, it has to be ensured that overfitting is avoided. Such long-lasting learning 
and data mining procedures can be prevented in the case of fuzzy logic.

Finally, and most importantly, the application of fuzzy logic procedures for the evaluation of exercises allows not only a reasonable, but also a direct and efficient integration of expertise knowledge and common strength training recommendations. Moreover, as the proposed work is natural and closer to human thinking, it is consequently easier to understand and use, promising hope for a convenient integration facility in machine-based routines, which can automatically assess the quality of the performed technique.

\subsection{Further Work and Outlook}

The future work will concentrate, on the one hand, on the optimization of the designed methods and the development of further intelligent techniques in strength training. For example, another opportunity for the definition of the completeness criteria might involve the consideration of additional determinants like the range of motion (ROM). Figure 8 illustrates a realization alternative for this kind of input variable in form of fuzzy sets, where a ROM (usually measured in ${ }^{\circ}$ ) of $90^{\circ}$ is considered to be optimal for a proper execution [30]. Such solutions, however, require the integration of further sensors such as goniometers, in order to be able to obtain the needed distance information.

The ultimate goal of the present study, on the other hand, is to integrate the proposed techniques into an already developed coaching framework [31], which would allow an immediate analysis and feedback on the execution.

\section{CONCLUSION}

The current research reviews the use of fuzzy logic techniques in the field of sport and illustrates their general applicability and promising implementation opportunities in the sportsspecific context. In particular, the paper proposes the development of fuzzy logic routines for analysis and evaluation purposes, demonstrated by an illustrative case study in strength training. The future scope of the approach will focus on the embedding of the presented methods into a server-based coaching framework for the individual and automated analysis of the gathered sensor data. This system integration would enable new facilities for the support of sportsmen and injury prevention, thereby also providing coaches with the opportunity to analyze and optimize the athletes' performances.

\section{REFERENCES}

[1] Łukasiewicz, J. 1920. "O logice trójwartościowej [On three-valued logic (in Polish)]”. Ruch filozoficzny, Vol. $5,170-171$.

[2] Zadeh, L.A. 1965. "Fuzzy Sets", Information and Control, Vol. 8, No. 1, 3, 338-353.

[3] Zadeh, L.A. 1965. Fuzzy sets and systems. In J. Fox, editor, System Theory. New York: Polytechnic Press, pp. 29-39.

[4] Boole, G. 1854. An Investigation of the Laws of Thought. Prometheus Books.

[5] Chua, S. C., Tan, W. C., Wong, E. K., and Koo, V. C. 2002. "Decision algorithm for pool using fuzzy system". In Proceedings of the International Conference on Artificial Intelligence in Engineering \& Technology, pp. 370-375.
[6] Chua, S. C., Tan, W. C., Wong, E. K., and Koo, V. C. 2004. "Decision algorithm for pool using fuzzy system (Part 2)". In Proceedings of the Second International Conference on Artificial Intelligence in Engineering \& Technology, pp. 691-697.

[7] Chua, S. C., Wong, E. K., and Koo, V. C. 2005. "Intelligent Pool Decision System Using Zero-Order Sugeno Fuzzy System". Journal of Intelligent and Robotic Systems, Vol. 44, No. 2, 161-186.

[8] Riley, J. 2005. Evolving fuzzy rules for goal-scoring behavior in a robot soccer environment, Doctoral Thesis, RMIT University, Melbourne, Australia.

[9] Jadon, R.S., Chaudhury, S., Biswas, K.K., Shakil, A. (2000). "A Fuzzy Theoretic Approach for Semantic Characterization of Video Sequences", ICVGIP'2000, pp. 73-78.

[10] Refaey, M.A., Essayed, K.M., Hanafy, S.M., and Davis, L.S. 2009. "Concurrent transition and shot detection in football videos using Fuzzy Logic". In Proceedings of the International Conference on Image Processing, pp. 4341-4344.

[11] Bartlett, R. 2003. "The science and medicine of cricket: an overview and update". Journal of Sports Sciences, Vol. 21, No. 9, 733-752.

[12] Bartlett, R. 2006. "Artificial intelligence in sports biomechanics: New dawn or false hope?". Journal of Sports Science and Medicine, Vol. 5, No. 4, 474-479.

[13] Curtis, K. M. 2009. "Cricket batting technique analyzer/trainer using fuzzy logic". In Proceedings of the $16^{\text {th }}$ International Conference on Digital Signal Processing, pp. 1056-1061.

[14] Curtis, K. M. 2010. "Cricket Batting Technique Analyzer/Trainer: A Proposed Solution using Fuzzy Set Theory to Assist West Indies Cricket". In Proceedings of the $9^{\text {th }}$ WSEAS International Conference on Artificial Intelligence, Knowledge Engineering and Data Bases, pp. 71-76.

[15] Singh, G., Bhatia, N., and Singh, S. 2011. "Fuzzy Logic Based Cricket Player Performance Evaluator". IJCA Special Issue on "Artificial Intelligence Techniques Novel Approaches \& Practical Applications”, pp. 11-16.

[16] Olaru, D., and Smith, B. 2002. "Fuzzy logic models for activity schedules". In Proceedings of $24^{\text {th }}$ Conference of Australian Institutes of Transportation Research, Sydney.

[17] Olaru, D., and Smith, B. 2003. "Modeling daily activity schedules with fuzzy logic", In Proceedings of the $10^{\text {th }}$ International Conference on Travel Behavior Research.

[18] Heller, M., and Witte, K. 2006. “A Dynamic Approach for Modeling and Simulation of Motor Unit Discharge Behavior Using Recurrent Fuzzy-Techniques". International Journal of Computer Science in Sport, Vol. 5, No. 1, 30-40.

[19] Mezyk, E., and Unold, 0. 2011. "Machine learning approach to model sport training. Computers in Human Behavior, Vol. 27, No. 5, 1499-1506.

[20] Hansen, M., 2006. "Recognizing of Movement Samples on the Basis of the Kinetics by Fuzzy Logic by the Example of Different Giant Swings". International 
Journal of Computer Science in Sport, Vol. 5, No. 2, 6467.

[21] Papić, V., Rogulj, N., and Pleština V. 2009. "Identification of sport talents using a web-oriented expert system with a fuzzy module". Expert Systems with Applications, Vol. 36, No. 5, 8830-8838.

[22] Novatchkov, H., and Baca, A. 2012. "Machine learning methods for the automatic evaluation of exercises on sensor-equipped weight training machines". In Procedia Engineering 9, ENGINEERING OF SPORT CONFERENCE 2012, Vol. 34, 562-567.

[23] Novatchkov, H., and Baca, A. 2013. "Artificial intelligence in sports on the example of weight training". Journal of Sports Science and Medicine, Vol. 12, No. 1, 27-37.

[24] Westcott, W. 2009. "ACSM strength training guidelines: Role in body composition and health enhancement". ACSM's Health \& Fitness Journal, Vol. 13, 14-22.

[25] Evans W. 1999. "Exercise training guidelines for the elderly". Medicine and Science in Sports and Exercise Vol. 31, No. 1, 12-17.
[26] Graham, J. F. 2008. Resistance exercise techniques and spotting. In Chandler, T. J., and Brown, L. E., Eds., Conditioning for Strength and Human Performance. Baltimore: Lippincott Williams \& Wilkins, pp. 182-236.

[27] Sivanandam, S.N., Sumathi, S., and Deepa, S.N. 2007. Introduction to Fuzzy Logic using MATLAB. Berlin, Heidelberg, New York: Springer.

[28] Ghosh, A., Meher, S.K., and Shankar, B.U. 2008. “A novel fuzzy classifier based on product aggregation operator", Pattern Recognition, Vol. 41, No. 3, 961-971.

[29] Kulkarni, U. V., and Shinde, S. V. 2013. "A Fuzzy Classifier based on Product and Sum Aggregation Reasoning Rule”. IJCA, Vol. 62, No. 5, 9-14.

[30] Housh, T. J., Cramer, J., Weir, J., Beck, T., and Johnson, G.O. 2008. Physical Fitness Laboratories on a Budget. Scottsdale, AZ: Holcomb Hathaway, pp. 108-116.

[31] Baca, A., Kornfeind, P., Preuschl, E., Bichler, S., Tampier, M., and Novatchkov, H. 2010. "A ServerBased Mobile Coaching System”. Sensors 2010, Vol.10, No. 12, 10640-10662. 